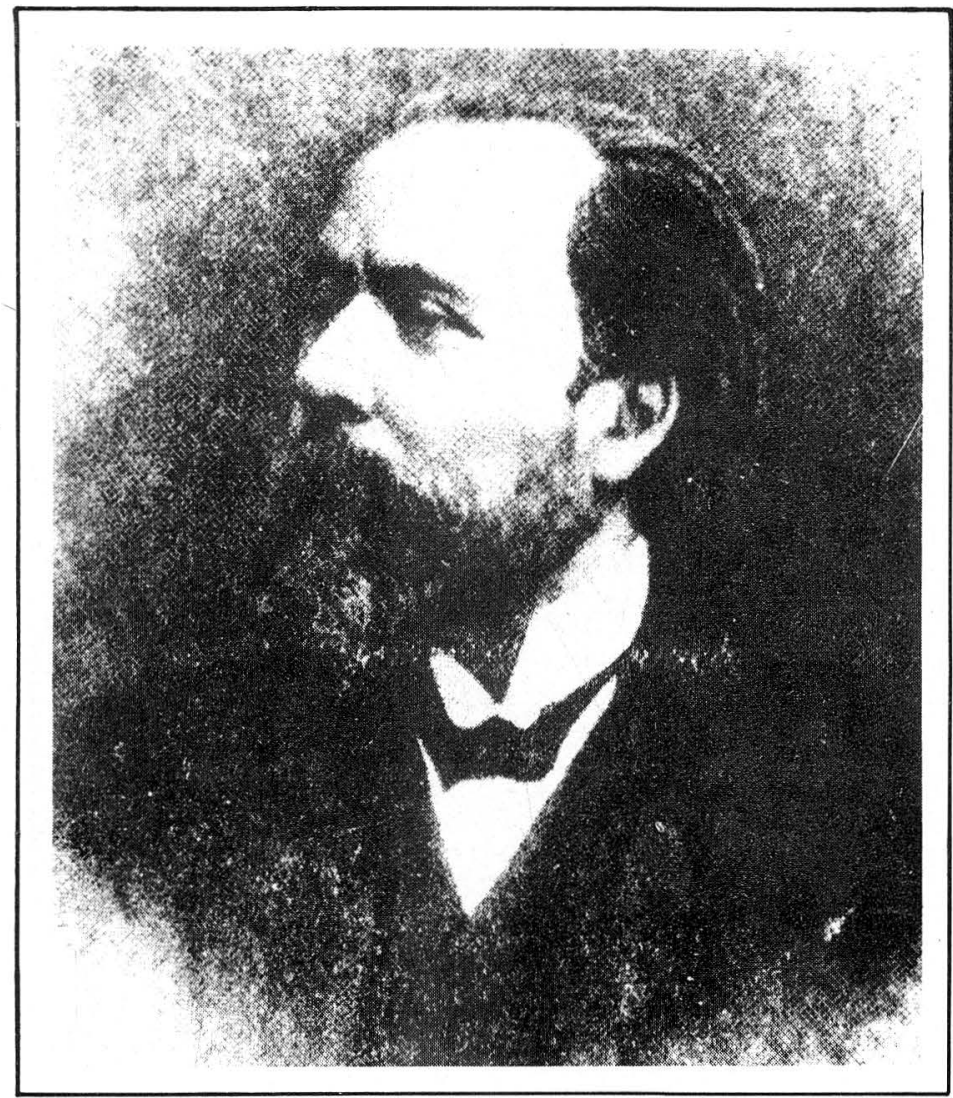

\title{
Historia de la Ginecobstetricia en Colombia
}

JUAN EVANGELISTA MANRIQUE

\author{
Por Fernando Sánchez Torres*
}

Promediando la segunda mitad del siglo XIX la cirugía comenzaba en Colombia a dar sus primeros pasos en firme, pues estaban de regreso al país algunos jóvenes médicos. que habían ido a Europa a ampliar sus conocimientos y a refrendar su título. Además, como ocurría en otras partes, los métodos anestésicos se habían implantado e inspiraban mayor confianza. La sepsis, en cambio, continuaba siendo el fantasma aterrador, no obstante la importante contribución que para combatirla había hecho el inglés Lister. Como es de suponer, la práctica de las grandes operaciones no era muy frecuente y sus indicaciones tenían que ser muy precisas y apremiantes como para que los pacientes corrieran los riesgos inherentes.

La cirugía ginecológica para entonces se reducía a unas pocas intervenciones, todas consideradas como "grandes operaciones": ovariectomía por tumores sintomáticos, histerectomías por la misma causa e histeropexias por prolapso genital insoportable. Quienes las practicaban eran cirujanos audaces, con entrenamiento teórico más que práctico. De ahí que sus actuaciones fueran tenidas como verdaderas hazañas y la supervivencia de las enfermas como algo milagroso. Además, a falta de instituciones dedicadas a esos menesteres, las operaciones solían llevarse a cabo en el mismo domicilio de las enfermas, lo cual le daba al procedimiento un ingrediente mayor de espectacularidad.

Con tal ambiente se encontró Juan Evangelista Manrique cuando regresó a Bogotá en 1886, luego de haber refrendado su título de médico en París en ese año, con la tesis

\footnotetext{
* Profesor Honorario de la Universidad Nacional de Colombia. Miembro Fundador de la Sociedad Colombiana de Historia de la Medicina.
} 
titulada "Etude sur l'operation d'Alexander", dedicada a los señores Angel y Rufino José Cuervo, y que le valió el "Lauro y Medalla de Bronce”. Dicha operación, propuesta por Alquié pero modificada y divulgada por Alexander, estaba destinada a corregir el prolapso del útero, acortando los ligamentos redondos. Esta circunstancia, la de haberse familiarizado con una entidad ginecológica para la presentación de su tesis, condujo a Manrique a convertirse, preferencialmente, en un cirujano ginecológico. Es seguro también que a esa inclinación contribuyera el haber sido espectador, y de pronto asistente o ayudante, de las demostraciones quirúrgicas, incluyendo las ginecológicas, que acostumbraban hacer, ante unos observadores alelados, los legendarios cirujanos que entonces campeaban en París: Faure, Leriche, Forgue, Pozzi, Doyen, Richelot . . .

Colocado en un terreno virgen, correspondió a Juan E. Manrique su exploración y conquista. Por eso se le considera acertadamente como uno de los pioneros de la cirugía ginecológica en Colombia. En ese terreno, antes de él, sólo figuraba Leoncio Barreto, quien practicó una ovariectomía en 1864. Razón le asistía cuando en 1888, al dar a conocer la intervención que llevó a cabo el 8 de septiembre de 1887, decía: "Tal es, honorables colegas, la historia de la primera operación de Alexander practicada en Colombia, y tal vez en la América Meridional, pues, como vosotros lo sabéis, me tocó a mí publicar el primer trabajo completo sobre esta operación, poco conocida en Francia y de empleo muy restringido en Inglaterra, Alemania y los Estados Unidos".

Los documentos que existen no permiten establecer con certeza a quien corresponde entre nosotros la primacía de la mayor y más delicada intervención ginecológica: la histerectomía abdominal. Yo me inclino a pensar que puede corresponder a Juan E. Manrique. Veamos por qué: en la sesión ordinaria de la Academia Nacional de Medicina, realizada el 4 de septiembre de 1908, el doctor Rafael Ucrós Durán declaró que el doctor Oscar Noguera la había ejecutado "21 años atrás” (es decir, en 1887) en Bogotá. Fuera de esta manifestación verbal no existe ninguna otra constancia. En el acta respectiva se consigna apenas que "el doctor Ucrós cree que fue la primera histerectomía practicada con buen éxito en Colombia y la presenta como un hecho que debe recogerse en la historia de la cirugía". Existe, en cambio, la certeza de que Manrique la llevó a cabo, sin buen éxito, es cierto, en Bogotá el día 6 de junio de 1887. Todos los detalles de este caso fueron referidos por el autor y recogidos en 1893 en la tesis de grado de Manuel Antonio Pérez. Vale la pena trascribir apartes que nos permiten darle todo su valor a tan importante acontecimiento:

“ (. . ) Al examinar el vientre, me sorprende su forma irregular. Del fondo del bacinete se levanta un tumor duro, liso, movible, el cual produce a la palpación la misma sensación que se experimenta cuando se explora el dorso de un feto a término; este tumor es más ancho hacia su extremidad superior que hacia la inferior. La parte superior llega hasta el hipocondrio derecho y el epigastrio; la parte inferior se pierde en la excavación pelviana ... De nuevo insistí en los peligros que esta operación ofrecía y la previne de que debía arreglar sus negocios y su conciencia, lo que muy poco impresionó a la enferma, quien a todo trance quería liberarse de los sufrimientos materiales, así como de las contrariedades morales que le ocasionaba el crecimiento de su vientre, el cual había dado origen a erradas interpretaciones de parte de las personas que la rodeaban. Anuncié también a la enferma que debía proveerse de un ajuar completamente nuevo y desinfectado por medio de la ebullición en una solución fenicada; y que debía buscar una casa que reuniera todas las condiciones higiénicas convenientes para no hacer inútil el sistema listeriano. 
"Después de haber buscado inútilmente una localidad conveniente en la ciudad, determiné visitar su casa de habitación, sobre el río del Arzobispo, y habiendo encontrado las mejores condiciones posibles para una laparotomía opté por dicha localidad....".

"Distribuido el trabajo y verificados todos los instrumentos y aparatos, procedimos a la operación, la cual fue retardada por la particular resistencia de la enferma a la cloroformización completa. El acto operatorio duró solamente tres horas y felizmente terminó a la 1 p.m. Las consecuencias inmediatas de la operación fueron las más felices".

A pesar de este optimismo inicial del cirujano, la paciente falleció dos días más tarde. La autopsia, practicada por los mismos que intervinieron en la operación "no mostró lesión capaz de explicar la muerte de la señorita R.’’

Alfonso Bonilla Naar afirma que entre nosotros la primera histerectomía vaginal fue practicada en Bogotá en 1888 por Juan E. Manrique. Considero que no fue así. La intervención a que se refiere el doctor Bonilla fue una miomectomía vaginal y no una histerectomía. Creo útil transcribir trozos de la descripción de esta operación, para poner de presente a qué atrevimientos conducía el justo temor que se le tenía a la cirugía abdominal.

Se trataba de una enferma de 32 años, virgen, que presentaba un gran tumor abdominal. En la casa de uno de los cuñados de la paciente, Juan E. Manrique, "ayudado por seis miembros de la cirugía menor", actuó así:

"Cloroformizada la enferma y colocada en la posición de litotomía, se cortó el hímen y se hizo una abundante irrigación vaginal. Se descubrió el cuello con dos valvas de Sims, colocadas a cada lado de la vagina y sostenidas por los mismos ayudantes que tenían las piernas ... Por medio de una aguja curva se pasó un fuerte hilo de seda en el labio anterior del cuello, con la ayuda del cual se fijó la matriz durante la parte intrauterina de la operación. Se procedió a la dilatación del cuello, primero con el dilatador de Pajot, pues el orificio del hocico era muy pequeño, luego con los dilatadores de Hegar, y en fin, para completarla hubo necesidad de debridar el cuello por medio de una incisión bilateral, practicada con las tijeras de Emmet. Terminada la dilatación fue fácil, con el dedo introducido en el útero, descubrir que en la cara anterior de la cavidad de la matriz sobresalía una parte del tumor. Debían existir otros tumores, pues el fondo de saco posterior estaba duro y el recto comprimido; además, el tumor, que llegaba hasta el borde costal, no se movía con la presión ejercida sobre la cara anterior de la matriz; sin embargo, el que existía en esta cara no solamente era el único accesible, sino que también debía ser el que determinaba la retención de orina. Estas consideraciones determinaron al cirujano a intentar la enucleación. Sirviéndose del dedo índice de la mano izquierda, como inductor, con la derecha se introdujo el largo bisturí abotonado de Sims, y se practicó una incisión longitudinal sobre la parte del tumor que sobresalía. Felizmente esta incisión cortó la cápsula en su parte más prominente y por ese punto fue posible separarla del tumor en una gran extensión. Las tracciones ejercidas sobre el tumor con la ayuda de las pinzas erinas implantadas sobre él, fueron infructuosas para extraerlo; y como ya hacía tres horas que la enferma estaba cloroformizada y sus fuerzas principiaban a decaer, se resolvió suspender la operación, no sin gran desencanto de todos los circunstantes, que esperaban ansiosos la salida del tumor diagnosticado. Después de hacer abundantes irrigaciones intrauterinas, se llenaron las cavidades uterina y vaginal con gasa yodoformada y se trasladó la enferma a su cama”. 
Doce días después, lapso este agitado y accidentado, la operada tuvo el parto de un tumor fibroso que pesó 800 gramos. La narración termina felizmente, pues se consigna que "tiempo después la paciente ha sido examinada por diferentes facultativos y ninguno ha encontrado el menor vestigio del tumor fibroso en su matriz".

Por supuesto que para haber llevado a cabo semejante hazaña se necesitaba tener el temple y la destreza de un gran cirujano. En efecto, Juan E. Manrique los tenía en grado sumo. Así lo confirmaron algunos de sus colegas contemporáneos. Abraham Salgar, por ejemplo, afirmó que "como cirujano poseía rara destreza, un sentido que podría llamarse artístico". Por su parte, el doctor Isaac Rodríguez, testigo de muchas de sus actuaciones, relató en 1904, en la Sociedad de Cirugía, lo siguiente: "Cuando apenas se iniciaba la época de la cirugía abdominal -finales del siglo- en El Campito de San José, en donde entusiasta oficiaba el doctor Manrique en beneficio de la ciencia y de los desheredados de la fortuna, se presentó una señora con un enorme tumor abdominal. Hecho el diagnóstico de fibroma uterino, se procedió a la histerectomía abdominal con todas las minuciosas exigencias de la cirugía moderna. Abierta la cavidad peritoneal, que él, con su jovialidad característica, llamaba entonces "una caja de sorpresas", en compañía de su distinguido colaborador e ilustre fundador del "Repertorio de Medicina y Cirugía", doctor José María Montoya, procedió a la enucleación de aquel voluminoso fibroma rodeado de adherencias; a pesar de una cuidadosa disección, fue interesado uno de los uréteres cerca de su extremidad vesical (aquíla inesperada complicación). Inmediatamente el hábil cirujano diseca el tubo ureteral y lo sutura convenientemente a la extremidad inferior de la herida abdominal. Por varios días sigue el curso de la orina hacia el exterior sin peligro alguno para la operada y más bien tuvimos la ocasión de observar el hecho clínico de que la orina aséptica que humedecía los labios saturados no impidió en manera alguna su rápida cicatrización.

"Salvado el primer peligro, nuestro infatigable cirujano, ansioso de volver el uréter a su puesto y de impulsar el interés científico de sus compañeros por la clínica quirúrgica de la Sociedad de Cirugía, convocó galantemente a todos sus miembros a fin de estudiar y decidir la intervención más acertada. Entonces se optó por la operación conocida con el nombre de Ureterocistoneostomía, la cual fue practicada cuando la enferma se halló en las mejores condiciones para soportar una segunda laparotomía.

“Con la mayor pulcritud y habilidad se llevó a efecto esta delicada operación, insertando por medio de finas y adecuadas suturas la extremidad ureteral directamente al punto más conveniente de la vejiga, lo cual dio por resultado en pocos días la curación de su interesante operada y la dulce satisfacción del éxito del cirujano”.

Por el testimonio anterior también podemos deducir que el doctor Manrique era un médico a quien gustaba transmitir y discutir sus experiencias, es decir, tenía vocación docente. Dado que la pasión política de la época le había negado la cátedra universitaria, en una policlínica fundada en 1895 por los doctores Lisandro Reyes y Eduardo Herrera, y posteriormente en su propia casa, dictaba conferencias sobre temas ginecológicos. El ya nombrado doctor Isaac Rodríguez, asistente a esas conferencias, recordaba así aquel insólito hecho: " ( . . ) quería con anhelo que todos sus compañeros amantes del deber participaran de sus conocimientos, y entonces, cuando apenas se iniciaban los estudios de Ginecología en nuestra Facultad, sin átomo de egoísmo, abrió en su casa un centro de conferencias magníficas en las que ostentaba su verbo poderoso, fácil y ameno, y su erudición sobresaliente entre los estudiantes y colegas que nos complacíamos en oirlo". Fue sólo hasta 1906 cuando se le nombró profesor de Clínica Quirúrgica.

La primera noticia que puede encontrarse sobre el tratamiento quirúrgico del em_barazo ectópico entre nosotros la proporciona Julio Pérez en su tesis de grado (1908). 
En ella recoge el autor una laparotomía seguida de salpingectomía por embarazo extrauterino roto, operación ejecutada en septiembre de 1901, en Bogotá, por los doctores, Luis Felipe Calderón y Juan E. Manrique Como el primero de los nombrados no figura en los anales de nuestra cirugía, es de soponer que quien operó a la paciente fue el segundo. Aunque el diagnóstico fue hecho oportunamente, los familiares se opusieron a la operación y sólo la aceptaron cuando la enferma se encontraba inconsciente por anemia aguda. "Dos horas después de terminada la operación -cuenta el doctor Calderónla enferma expiraba en medio de las más acres y agresivas manifestaciones de hecho y de palabra de los que la rodeaban, sin que esto alcanzara a entibiar en nosotros la profunda satisfacción del deber cumplido, que a mi ilustre compañero y a mí nos dejaba esa intervención".

Tal como expresé antes, Juan E. Manrique regresó de París con ideas innovadoras en el terreno de la ginecología. Considero que él, junto con Rafael Ucrós Durán, fundador de la cátedra ginecológica en la Universidad Nacional en 1903, fueron quienes le dieron personalidad a la especialidad en Colombia.

Dos ejemplos pueden servir para aceptar el avanzado criterio que en cuestiones ginecológicas tenía Manrique. Hélos a continuación:

1. A finales de siglo el tratamiento de la peritonitis era exclusivamente médico. Se prescribían tónicos tales como el alcohol en forma de vinos generosos, la cafeína en inyección hipodérmica y el oxígeno en inhalaciones. En caso de náuseas y vómitos se daba champaña. Se creía también, siguiendo las recomendaciones de Widal, que la administración de opio a dosis elevadas -30 centigramos al día-junto con antisépticos intestinales producía la curación de la peritonitis supuradas. Para algunos intervencionistas - que eran los menos- la laparotomía estaba indicada si existían manifestaciones de "peritonitis con derrame". El drenaje y la limpieza, y hasta la histerectomía en los casos severos eran - a juicio de Manrique- una conducta "que no da lugar a una sola objeción". Para entonces, 1906, apenas se conocían tres casos manejados en Bogotá con ese criterio: dos a manos del doctor Manrique, uno de ellos con muerte de la enferma, y el tercero operado por el doctor Zoilo Cuéllar Durán, también con terminación fatal.

2. Las ulceraciones crónicas del cervix se aconsejaba que fueran tratadas con la resección de la mucosa, la amputación del cuello o la escarificación a hierro rojo. No obstante, uno de los métodos más usados era el propuesto por los doctores Richelot, padre e hijo, en París , con el cáustico llamado "Neofilhos". Su acción debía de ser muy intensa,pues una sola aplicación bastaba para formar escara y algunas veces estenosis cervical, tal como lo aseverara Manrique en una de sus conferencias de Clínica Quirúrgica: "Debo hacer - decía- una corrección a mi maestro Richelot: el cáustico Neofilhos produce estenosis del cuello, a veces fácilmente dilatable".

Juan Evangelista Manrique nació en 1861 en la hacienda "La Herrera", situada en el municipio de Bojacá, Cundinamarca. Cursó estudios de literatura y filosofía en el acreditado colegio de don Luis M. Cuervo hermano mayor del ilustre filólogo Rufino José Cuervo. Allí fue su condiscípulo José Asunción Silva, más tarde príncipe de la poesía colombiana. En 1882 se graduó de médico en la facultad de medicina de la Universidad Nacional. Al poco tiempo viajó a París donde, como ya registramos, refrendó su título con todos los honores. Durante los años de 1883 y 1884 refrendó también su 
amistad con Silva quien, en plan de negocios, vivía en la Ciudad Luz. Precisamente, fue nuestro médico quien en Bogotá, en 1896, y a instancias del interesado, le señaló al poeta con un lápiz dermográfico el sitio donde latía la punta de su corazón. Al día siguiente lo encontraron muerto, víctima de un pistoletazo que él mismo se dio, exactamente en el punto marcado por Manrique.

En 1886 regresó a Bogotá, donde ejerció, con singular y explicable éxito, por algo más de veinte años. Como era un hábil cirujano general, le correspondió practicar variadas y difíciles intervenciones, como una trepanación hecha en 1888 por "traumatismo craneano y herida de la meningea media”. Durante la Guerra de los Mil Días prestó sus servicios en las ambulancias de la Cruz Roja, organizadas por destacados médicos radicados en Bogotá, para atender a los ejércitos revolucionarios. Fue miembro de número de la Academia Nacional de Medicina, miembro fundador del Club Médico, del Hospital San José y de la Sociedad de Cirugía, de la cual fue su primer presidente y redactor de sus estatutos y reglamentos.

En el desempeño de funciones diplomáticas, Juan Evangelista Manrique murió en San Sebastián, España, el 13 de octubre de 1914. El maestro Guillermo Valencia, orador durante el homenaje que se le rindió en el primer aniversario de su muerte, dijo de él; "La excelencia del médico respaldaba también la calidad del cirujano. La intervención a que él concurriera fue siempre sólidamente motivada, y en ese a modo de combate en que, como en los bélicos, la ciencia ordena y el hierro decide, Manrique fue el experto que conjuró peligros, sorteó dificultades y transformó en provecho ingente lo imprevisto".

\section{BIBLIOGRAFIA}

Academia Nacional de Medicina. Acta de la sesión ordinaria del 4 de septiembre. Bogotá, 1908.

BONILlA-NAAR, A. "Precursores de la Cirugía en Colombia". Ed. Antares, Bogotá, 1954.

CIFUENTES, M.A. "Contribución al estudio de la infección puerperal". Tesis de grado. Imprenta "La Luz”, Bogotá, 1906.

MANRIQUE, J.E. "Etude sur l'operation D'Alexander", G. Steinheil, Editeur. París, 1886.

MANRIQUE, J.E. "Prolapsus del útero. Operación de Alexander". Revista Médica, Bogotá, 12: 216, 1888.

MANRIQUE, J.E., HERRERA, J.D. "Fibromioma intersticial”. Revista Médica, Bogotá, 127: 104, 1888.

MANRIQUE, J.E. "Prolapsus del útero acompañado de fenómenos locales y generales incompatibles con los trabajos habituales.
Operación de Alexander. Curación”. Revista Médica, Bogotá, 130: 215,1888 ,

MANRIQUE, J.E., MONTOYA, J.M. "Heridas del útero en el curso de las operaciones abdominales". Repertorio de Medicina y Cirugía. Bogotá, 1: 311, 1910.

MUÑOZ, L. "Historia del Hospital San José, 1902-1956". Imprenta del Banco de la República, Bogotá, 1958.

PEREZ, J. "El embarazo extrauterino y su tratamiento". Tesis de grado. Imprenta Eléctrica, Bogotá, 1908.

PEREZ, M.A. "Contribución al estudio de la cirugía abdominal en Colombia". Tesis de grado. Casa Editorial de J.J. Pérez, Bogotá, 1893 .

RUEDA, M. "Tratamiento de la metritis cervical crónica por el cáustico Neofilhos". Tesis de grado. Imprenta Nacional, Bogotá, 1908.

VALENCIA, G. "Discursos". Instituto Caro y Cuervo. Biblioteca Colombiana VIII, Bogotá, tomo II, p. 46, 1973. 